\title{
Gram staining in Microbiology for second-year MBBS students: Comparative study of student scores in Objective Structured versus Traditional Practical Examination
}

\author{
Milind R. Ubale ${ }^{1}$, Rajani P. Tore ${ }^{2}$, Sundaram Kartikeyan ${ }^{3}$, \\ Aniruddha A. Malgaonkar ${ }^{3}$ \\ ${ }^{I}$ Microbiology Department, Rajiv Gandhi Medical College, Kalwa, Thane, Maharashtra, India \\ ${ }^{2}$ Microbiology Department, Indira Gandhi Government Medical College, Nagpur, Maharashtra, India \\ ${ }^{3}$ Community Medicine Department, Rajiv Gandhi Medical College, Kalwa, Thane, Maharashtra, India
}

\begin{abstract}
This cross-sectional comparative study on Gram staining was conducted in a medical college in Maharashtra, Western India. Fourth semester MBBS students (n=90) with prior exposure to traditional practical examination in Microbiology were randomized by lottery method and divided into three groups of 30 students each. Students in Groups 1 \& 3 were evaluated by traditional practical examination. Group -2 students rotated through a 3-station objective structured practical examination wherein the pre-validation of the checklist and questionnaire was done by university-approved senior teachers. There were significant differences in the marks scored by students in Groups $1 \& 3(Z=3.818 ; p<0.002)$ though they were exposed to the same pattern of traditional practical examination. This could be ascribed to inadequate uniformity in the allocation of marks. The standard deviation of scores for Group-2 (objective structured practical examination group) was the least among the three groups indicating consistency in allocation of marks.
\end{abstract}

Keywords : Gram stain, Objective Structured Practical Examination

\section{Introduction}

The Objective Structured Practical Examination (OSPE) is a reliable objective and structured method of skill-based assessment by direct observation of the student's performance in an adaptable examination set-up that is based on a circuit of laboratory "stations." [1-5] While the traditional practical examination (TPE) primarily lays emphasis on the "knows" and "knows how" aspects (the first and second levels of Miller's Framework of development of competencies), the OSPE assesses the third level (the "shows how" level) that stresses on evaluation of performance of specific psychomotor skills. [6] For a reliable skill-based assessment, student performance has to be evaluated across a range of situations. [1]

Traditional practical examinations are chiefly subjective and largely examine the cognitive (knowledge) component rather than the psychomotor (competence) component. As compared to traditional practical examinations, the OSPE appraises a range of competencies [7,8], measures practical psychomotor skills better, eliminates subjectivity, [7] has a wider discrimination index and helps students to grasp various components of competencies and also obtain feedback. [9] The barriers to use of OSPE include its labourintensive nature, snags in maintaining uniform difficulty levels, and observer fatigue. [10] Currently, OSPE has been introduced in select Indian universities [7,11] but there are no national-level guidelines on conducting OSPE. The Gram staining technique was selected for OSPE in the present study because this is categorized in the "must know" component of the university syllabus for the subject of Microbiology for the second-year MBBS course and carries five marks in the university practical examination. Furthermore, Gram staining technique tests the psychomotor skills of the student.

\section{Objectives}

The aim of the present study was to compare scores obtained by students in OSPE with that obtained in TPE.

\section{III. materials and methods}

3.1. Type of study: Cross-sectional comparative study

3.2. Study setting: Microbiology laboratory of a medical college in Maharashtra, Western India

3.3. Participants: Fourth semester (second-year) MBBS students

3.4. Inclusion criteria: All fourth semester students, of either sex, who gave written informed consent to participate in the study.

3.5. Exclusion criteria: Those who did not give written informed consent.

3.6. Procedure: After obtaining permission from the Institutional Ethics Committee, all fourth semester students (who were already exposed to TPE) were explained about the study and written informed consent was 
obtained. Detailed instructions were given to students regarding the pattern of examination and the course to be covered. Students $(n=90)$ were randomized by lottery method and divided into three groups of 30 students each. Group-1 \& Group-3 students were evaluated by TPE and Group-2 students by OSPE. (Fig. 1)

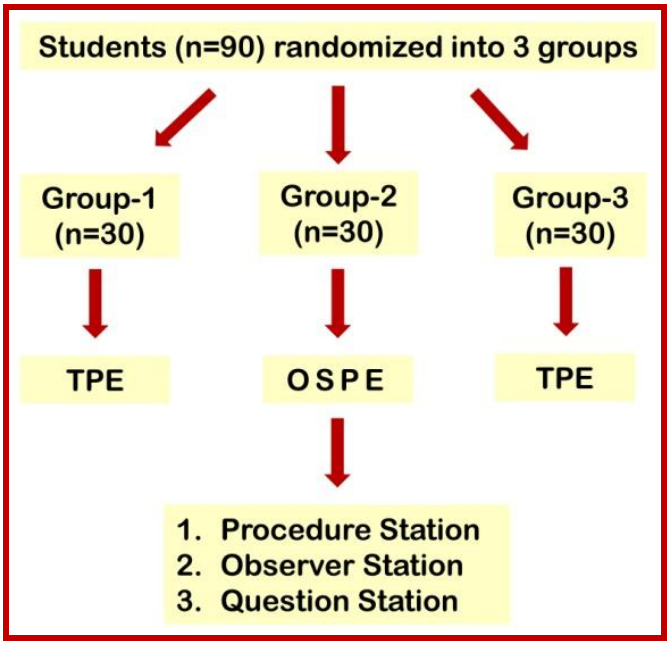

Fig. 1: Diagrammatic representation of the study procedure

3.7. Structuring OSPE: Three types of OSPE stations were included for Group-2 students - (a) Procedure station: the students were required to perform Gram staining procedure; the checklist at the procedure station comprised marks for proper staining technique, hand not getting stained, proper cleaning of work table after procedure and discard of waste, and questions related to principle of staining and the procedure; (b) Observation Station: the students observed a focused field under oil immersion lens of the microscope and recorded their observations; the components of the checklist at the procedure station were adjustment of the mirror, diaphragm and condenser of the microscope, time taken to focus smear, proper staining, interpretation and question related to microscopy, particularly oil immersion. (c) Question station: a pre-validated questionnaire was answered by the student at this station. The total time for OSPE was pre-determined based on the sum of the expected time at the three stations. The observer in the procedure station, who was provided with a pre-validated checklist, appraised the students while they performed the procedure and awarded marks for each step. Pre-validation of the checklist and questionnaire was done by university-approved senior teachers.

3.8. Statistical analysis: Data were presented as percentages, mean and standard deviation (SD) and statistically analyzed using EpiInfo Version 7.0 (public domain software package from the Centers for Disease Control and Prevention, Atlanta, GA, USA). Confidence interval (CI) was stated at 95\% interval as: [Mean-(1.96)*Standard Error)] - [Mean+(1.96)*Standard Error)]. The standard error of difference between two means was calculated. Statistical significance was determined at $\mathrm{p}<0.05$.

\section{Results And Discussion}

4.1. Mean scores: A total of 90 students participated in the study. The gender-wise differences in scores were not statistically significant. The standard deviation of scores for Group-2 (OSPE group) was the least among the three groups indicating that the scatter of marks about the mean was lower in the OSPE group (Table 1). This implied better consistency of the marking system for the OSPE group. Statistically significant differences were observed between the scores obtained by Groups $1 \& 2(\mathrm{Z}=2.435 ; \mathrm{p}=0.0146)$, Groups 2 \& 3 ( $\mathrm{Z}=2.150$; $\mathrm{p}=0.0316)$ and Groups $1 \& 3(\mathrm{Z}=3.818 ; \mathrm{p}<0.002)$.

Though all students who participated in the study had prior exposure to TPE, there were statistically significant differences $(Z=3.818 ; p<0.002)$ in the marks scored by students in Groups 1 \& 3 who were exposed to TPE in the present study, which could be ascribed to inadequate uniformity in the allocation of marks.

Table 1: Mean scores obtained by the 3 groups

\begin{tabular}{|l|c|c|c|}
\hline & Group-1 & Group-2 & Group-3 \\
\hline Mean & 3.44 & 3.81 & 4.15 \\
\hline SD & 0.70 & 0.45 & 0.74 \\
\hline 95\% CI & $3.19-3.69$ & $3.65-3.97$ & $3.89-4.41$ \\
\hline
\end{tabular}

$\mathrm{SD}=$ Standard deviation; $\mathrm{CI}=$ Confidence interval

Usually, in TPE, the aspects of the exercise, the number of questions asked to each student, the type of question asked and the time spent may vary across examiners even for each student. This creates a discrepancy 
in allocation of marks and is subject to examiner bias. A study from Saudi Arabia has reported considerable improvement in the mean scores in OSPE. [12] However, other studies have reported no significant difference between the scores obtained in conventional practical examination and that obtained in OSPE. $[13,14]$

4.2. Boxplot of scores: The maximum score ( 5 marks) and the minimum score of 2.25 and 2.5 in Group- 1 and Group-3, respectively, indicate high level of variation (dispersion) in marks in the TPE groups. (Fig. 2)

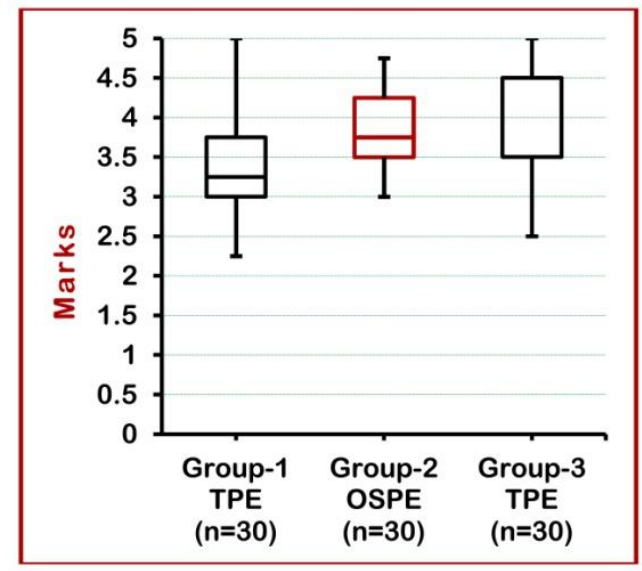

Fig. 1: Boxplot of scores obtained by the three groups

The median score was 3 and 3.5 in Group-1 and Group-3 (both TPE groups), respectively. The median mark (3.75) of the OSPE group is at the same level of third quartile of Group-1. The median of Group-3 has merged with the third quartile, which could be attributed to over-marking.

The OSPE model is highly objective since all examinees are exposed to a pre-determined set of questions that examine a range of competencies. Though OSPE is not a flawless tool for appraisal, it brings about a considerable improvement in student assessment and eliminates subjectivity. [7] Researchers have reported that the outcome of OSPE was reduction in total time for practical examination, uniformity in student assessment, decrease in the stress of students [11] and reduction in examiner bias. [15] However, OSPE has not yet been implemented for practical examinations for the second-year MBBS course in the subject of Microbiology since it is not yet recommended as an evaluation tool by the Maharashtra University of Health Sciences.

4.3. Limitations: The results of this study cannot be generalized due to its small sample size and low marking range of $0-5$. This marking range was retained at the same level recommended for university examination. Crossing over of groups was also not carried out due to time constraints.

\section{Conclusion}

As compared to TPE, the 3-station OSPE study for Gram staining showed improvement in the mean scores with reduction in deviation of marks about the mean implying increased consistency while awarding marks. On the similar pattern of the 3-station OSPE in the present study, which served the purpose of formative assessment for Gram staining procedure, an Extended OSPE (EOSPE) can be designed to include all the components of practical evaluation as prescribed in the curriculum and each aspect can be assigned the desired weights in the marking system.

\section{References}

[1] S. Smee, ABC of learning and teaching in medicine: Skill based assessment, BMJ, 326(7391), 2003, $703-706$.

[2] R. M. Harden, What is an OSCE?, Med Teacher, 10(4), 1988, 19-22.

[3] R. M. Harden and F. A. Gleeson, ASME Medical Education Booklet No. 8: Assessment of clinical competence using an objective structured clinical examination (OSCE), Med Educ, 13, 1979, 41-54.

[4] R. M. Harden, How to assess clinical competence: An overview, Med Teacher, 1(8), 1979, 289-296.

[5] R. M. Harden, M. Stevenson, D. W. Wilson, and G. M. Wilson, Assessment of clinical competencies using objective structured clinical examination, Br Med J, 1(5955), 1975, 447-451.

[6] G. E. Miller, The assessment of clinical skills/competence/performance, Acad Med, 65 (9 Suppl), 1990, S63-67.

[7] J. Charles, A. Janagond, Thilagavathy, Rajendran, Ramesh, and Vidhya, Evaluation By OSPE (Objective Structured Practical Examination) - A good tool for assessment of medical undergraduates - A study report from Velammal Medical College, Madurai, Tamilnadu, India, IOSR-JRME, 6(3), 2016, 1-6.

[8] R. K. Reznick, S. Smee, J. S. Baumber, R. Cohen, A. Rothman, D. Blackmore, et al., Guidelines for estimating the real cost of an objective structured clinical examination, Acad Med, 68(7), 1993, 513-517. 
[9] P. Gupta, P. Dewan, and T. Singh, Objective Structured Clinical Examination (OSCE) Revisited, Med Educ, 47(11), 2010, 911920.

[10] B. Gitanjali, The other side of OSPE, Indian J Pharmacol, 36(6), 2004, 388-389.

[11] R. R. Abraham, R. Rao, S. Kamath, and A. Kamath, A trial of the objective structured practical examination in physiology at Melaka Manipal Medical College, India, Adv Physiol Educ, 33, 2009, 21-23.

[12] A.S. Dissanayake, B. A. Ali, and U. Nayar, The influence of the introduction of objective structured practical examinations in physiology on student performance at King Faisal University Medical School, Med Teach, 12, 1990, $297-304$.

[13] S. D. Malhotra, K. N. Shah, and V. J. Patel, Objective structured practical examination as a tool for the formative assessment of practical skills of undergraduate students in pharmacology, J Educ Health Promot, 2, 2013, 53.

[14] A.S. Mahajan, N. Shankar, O. P. Tandon, The comparison of OSPE with conventional physiology practical assessment, Med Sci Educator, 14(2), 2004, 54-57.

[15] D. Kundu, H. N. Das, G. Sen, M. Osta, T. Mandal, and D. Gautam, Objective structured practical examination in Biochemistry: An experience in Medical College, Kolkata, J Nat Sci Biol Med, 4(1), 2013, 103-107.

\footnotetext{
Milind R. Ubale. "Gram staining in Microbiology for second-year MBBS students: Comparative study of student scores in Objective Structured versus Traditional Practical Examination." IOSR Journal of Research \& Method in Education (IOSR-JRME) 7.4 (2017): 01-04. 